Ferrata Storti Foundation

\title{
Human leukocyte antigen class II expression is a good prognostic factor in adult T-cell leukemia/lymphoma
}

Haematologica 2019

Volume 104(8):1626-1632

\section{Correspondence:}

KOICHI OHSHIMA

ohshima_kouichi@med.kurume-u.ac.jp

Received: August 28, 2018.

Accepted: January 9, 2019.

Pre-published: January 10, 2019.

doi:10.3324/haematol.2018.205567

Check the online version for the most updated information on this article, online supplements, and information on authorship \& disclosures: www.haematologica.org/content/104/8/1626

(C)2019 Ferrata Storti Foundation

Material published in Haematologica is covered by copyright. All rights are reserved to the Ferrata Storti Foundation. Use of published material is allowed under the following terms and conditions:

https://creativecommons.org/licenses/by-nc/4.0/legalcode. Copies of published material are allowed for personal or internal use. Sharing published material for non-commercial purposes is subject to the following conditions:

https://creativecommons.org/licenses/by-nc/4.0/legalcode, sect. 3. Reproducing and sharing published material for commercial purposes is not allowed without permission in writing from the publisher.

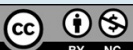

\author{
Mai Takeuchi, ${ }^{1}$ Hiroaki Miyoshi, ${ }^{1}$ Naoko Asano, ${ }^{2}$ Noriaki Yoshida, ${ }^{1,3}$ Kyohei \\ Yamada, ${ }^{1}$ Eriko Yanagida, ${ }^{1}$ Mayuko Moritsubo, ${ }^{1}$ Michiko Nakata, ${ }^{1}$ Takeshi \\ Umeno, ${ }^{1}$ Takaharu Suzuki, ${ }^{1}$ Satoru Komaki, ${ }^{1}$ Hiroko Muta, ${ }^{1}$ Takuya Furuta, ${ }^{1}$ \\ Masao Seto ${ }^{1}$ and Koichi Ohshima ${ }^{1}$ \\ ${ }^{1}$ Department of Pathology, Kurume University School of Medicine, Kurume, Fukuoka; \\ ${ }^{2}$ Department of Molecular Diagnostics, Nagano Prefectural Shinshu Medical Center, \\ Suzaka, Nagano and ${ }^{3}$ Department of Clinical Studies, Radiation Effects Research \\ Foundation, Hiroshima, Japan
}

\section{ABSTRACT}

A ttenuated human leukocyte antigen (HLA) class I expression is implicated as a major immune escape mechanism in several types of tumor. We previously reported that HLA class I/ $\beta 2$ microglobulin and programmed death ligand-1 expression are prognostic factors in adult T-cell leukemia/lymphoma. A recent report suggested that HLA class II expression is also an important prognostic factor for the clinical outcome of programmed death-1 blockade therapy in recurrent/refractory Hodgkin lymphoma. This prompted us to evaluate HLA class II expression in adult T-cell leukemia/lymphoma and to compare the findings with the patients' clinicopathological features. Of the 132 biopsy specimens examined from newly diagnosed patients, lymphoma cells were positive for HLA class II expression in 44 patients (33.3\%), whereas programmed death ligand-1 expression was observed on neoplastic cells from nine patients $(6.9 \%)$ and on stromal cells in the tumor microenvironment in 83 cases $(62.9 \%)$. HLA class II-positive cases showed a significantly better overall survival compared to the HLA class II-negative cases $(P<0.0001)$. Patients positive for HLA class II and programmed death ligand- 1 microenvironmental expression had significantly better prognosis than the other groups $(P<0.0001)$. HLA class II-positive and HLA class II-negative groups also showed a significant difference in complete remission rate $(P=0.0421)$, HLA class $I / \beta 2$ microglobulin expression $(P=0.0165)$, and the number of programmed death-1-positive tumor infiltrating cells $(P=0.0020)$. HLA class II expression was a prognostic factor for overall survival both in univariate and multivariate analyses $(P<0.0001$ and $P=0.0007$, respectively). Our study reveals that HLA class II is a novel prognostic factor in adult T-cell leukemia/lymphoma.

\section{Introduction}

Adult T-cell leukemia/lymphoma (ATLL), caused by human T-cell lymphotropic virus type 1 (HTLV-1), is an aggressive hematopoietic malignancy with a poor prognosis. HTLV-1 carriers are common in coastal areas of south-western Japan, and approximately 2-5\% of carriers develop ATLL in their lifetime..$^{1,2}$ According to the Shimoyama classification, there are four clinical subtypes of ATLL, namely acute, lymphoma, chronic, and smoldering. ${ }^{3}$ The acute and lymphoma types are typically associated with a poor prognosis. ${ }^{3}$ The chronic and smoldering types show better prognosis than the acute and lymphoma types. ${ }^{3}$ However, half of the cases of chronic ATLL have been reported to transform into leukemia. ${ }^{4}$

Programmed cell death ligand-1 (PD-L1) is expressed on various tumors and is considered to contribute to tumor immune escape. ${ }^{5.9}$ We previously reported that PD-L1 expression in tumor cells and stromal cells of the tumor microenvironment are prognostic factors in ATLL. ${ }^{10}$

Presentation of neoantigens by human leukocyte antigen (HLA) class I is required for $\mathrm{CD}^{+}$cytotoxic $\mathrm{T}$ cells to attack tumor cells. However, the expression of HLA class I and/or its component $\beta 2$ microglobulin $(\beta 2 \mathrm{M})$ is frequently reduced or lost 
in some tumors, which plays an important role in immune escape and survival of tumors. ${ }^{11}$ Kataoka et al. found that more than half of ATLL patients had alterations in HLA class I genes. ${ }^{12}$ We have previously reported that ATLL expressing HLA class $\mathrm{I} / \beta 2 \mathrm{M}$ had a good clinical outcome. ${ }^{13}$

Programmed cell death-1 (PD-1) blockade therapy is effective in various recurrent/refractory tumors. PD- 1 is expressed on activated T cells and binds to PD-L1 on tumor cells. Binding of PD-L1 to PD-1 suppresses production of cytokines and inactivates T cells. ${ }^{14,15}$ HLA class I plays a key role in PD-1 blockade therapy because activated $\mathrm{T}$ cells attack tumor cells by recognizing neoantigens presented by HLA class I on tumor membranes. In fact, an interaction between neoantigens and neoantigen-specific $T$ cells via HLA class I has been reported in patients with malignant melanoma who received PD-1 blockade therapy. ${ }^{16}$

It has been hypothesized that tumors lacking HLA class I and/or $\beta 2 \mathrm{M}$ could be resistant to PD-1 blockade therapy. ${ }^{17}$ However, a favorable clinical response to PD-1 blockade has been observed in patients with Hodgkin lymphoma, who usually lack HLA class I expression due to the loss-of-function mutation of $\beta 2 \mathrm{M}^{18,19}$ From the standpoint of immunotherapy, these results are indicative that other immunological mechanisms exist, at least among patients with Hodgkin lymphoma.

HLA class II is expressed on antigen-presenting cells including B cells, dendritic cells, and macrophages. HLA class II presents antigens to $\mathrm{CD}^{+} \mathrm{T}$ cells.

HLA class II expression has been reported to be associated with a favorable prognosis in squamous cell carcinoma of the larynx, colorectal cancer, and diffuse large B-cell lymphoma ${ }^{20-22}$ In contrast, HLA class II expression was not significantly associated with prognosis in malignant melanoma, Hodgkin lymphoma, and lung cancer treated with standard chemotherapies. ${ }^{23-25}$

Roemer et al. recently reported that HLA class II expression is more important than HLA class I expression for PD-1 blockade therapy in recurrent/refractory Hodgkin lymphoma. ${ }^{26}$ Although the precise mechanism remains unknown, it has been suggested that an interaction between HLA class II-expressing lymphoma cells and $\mathrm{CD}^{+} \mathrm{T}$ cells could contribute to tumor immunity in Hodgkin lymphoma.

The prognosis of ATLL patients treated with standard chemotherapy remains poor. PD-1 blockade could be a good therapeutic strategy; however, a clinical trial showed that PD-1 blockade in ATLL patients induced disease progression. ${ }^{27}$ For a better understanding of tumor immunity associated with PD-L1/PD-1 in ATLL, we examined the clinico-pathological effect of HLA class II expression in ATLL and the correlation of HLA class II to HLA class $\mathrm{I} / \beta 2 \mathrm{M}$ and PD-L1/PD-1 expression.

\section{Methods}

\section{Patients and samples}

All patients included in this study were the same as those in our previous reports. ${ }^{10,13}$ Fifty-nine patients were derived from the International Peripheral T-Cell Lymphoma Project, and 73 were newly diagnosed with ATLL in Kurume University between 2006 and $2012 .^{28} \mathrm{~A}$ tissue microarray including 132 samples in formalinfixed paraffin-embedded blocks was created. The tissue microarray specimens used in this study were the same as those examined in our previous studies. ${ }^{10,13}$ The use of patients' specimens and clin- ical data was approved by the Research Ethics Committee of Kurume University and was carried out in accordance with the Declaration of Helsinki.

\section{Immunohistochemistry}

Immunohistochemistry was performed as previously described. ${ }^{10,13}$ The following antibodies were used: HLA class II (CR3/43 M0775, DAKO, Glostrup, Denmark), HLA class I (EMR85; Abcam, Tokyo, Japan), ß2M (HPA006361; Sigma-Aldrich, Tokyo, Japan), PD-L1 (EPR1 161(2); Abcam), and PD-1 (NAT105, Abcam).

As in our previous studies, PD-L1 expression was determined on neoplastic cells (nPD-L1) and microenvironmental stromal cells (miPD-L1). nPD-L1 was considered positive when $50 \%$ or more neoplastic cells were positive for PD-L1.$^{10}$ miPD-L1 were distinguished from nPD-L1 by irregular shaped morphology, low nuclear/cytoplasmic ratio, and nuclei without atypia (Online Supplementary Figure S1A, S1B $\cdot .^{10}$ miPD-L1 was defined as positive in patients with ten or more $\mathrm{PD}-\mathrm{L}^{+}$non-neoplastic stromal cells per high power field..$^{10}$ Other markers, including HLA class II, were considered positive when $30 \%$ or more tumor cells were positive. $\mathrm{PD}-1^{+}$tumor-infiltrating lymphocytes were detected as small lymphocytes without atypia and were distinguished from PD-1 tumor cells (Online Supplementary Figure S1C, S1D). PD-1+ tumorinfiltrating lymphocytes were counted in five representative highpower fields, and the average number for each sample was calculated as previously reported. ${ }^{10}$

\section{Statistical analysis}

Clinico-pathological characteristics were compared by the $\chi^{2}$ test or Fisher two-sided exact test. Wilcoxon rank sum test was performed to compare age and $\mathrm{PD}-1^{+}$tumor-infiltrating lymphocyte counts. Overall survival was defined as the period between the day of diagnosis and the day of death or the day of last follow up. Overall survival was analyzed by the Kaplan-Meier method, and the log-rank test was performed to determine significant differences. Univariate and multivariate Cox proportional regression models were used to examine the proposed prognostic factors. All statistical analyses were performed using JMP version 11.0. $P$ values $<0.05$ were considered statistically significant.

\section{Results}

\section{Clinico-pathological characteristics}

The participants' clinical information is summarized in Table 1 . The median age of the participants was 67.5 years (range, 35-90), and the male to female ratio was 75:57. Seventy-eight of 126 (61.9\%) patients had a high International Prognostic Index (high or high-intermediate), and 50/129 (38.8\%) patients had high scores in the Japan Clinical Oncology Group Prognostic Index (JCOG-PI). ${ }^{29}$

According to the Shimoyama classification, most cases were acute type $(53 / 108,49.1 \%)$ or lymphoma type (52/108, $48.1 \%)$. Only three cases $(2.8 \%)$ were smoldering ATLL and no cases of chronic ATLL were included in this study. Most patients received chemotherapy (115/131, $87.8 \%)$; radiation $(14 / 129,10.9 \%)$ and allogenic stem cell transplantation $(17 / 129,13.2 \%)$ were chosen in fewer cases. The chemotherapy regimens were not significantly different between the HLA class II-positive and negative groups (Online Supplementary Table S1). No PD-1 blockade therapy was given. Of the 115 patients, 32 (27.8\%) achieved a complete remission. The median follow-up period was 10.96 months (range, 0.03-114.8 months). 

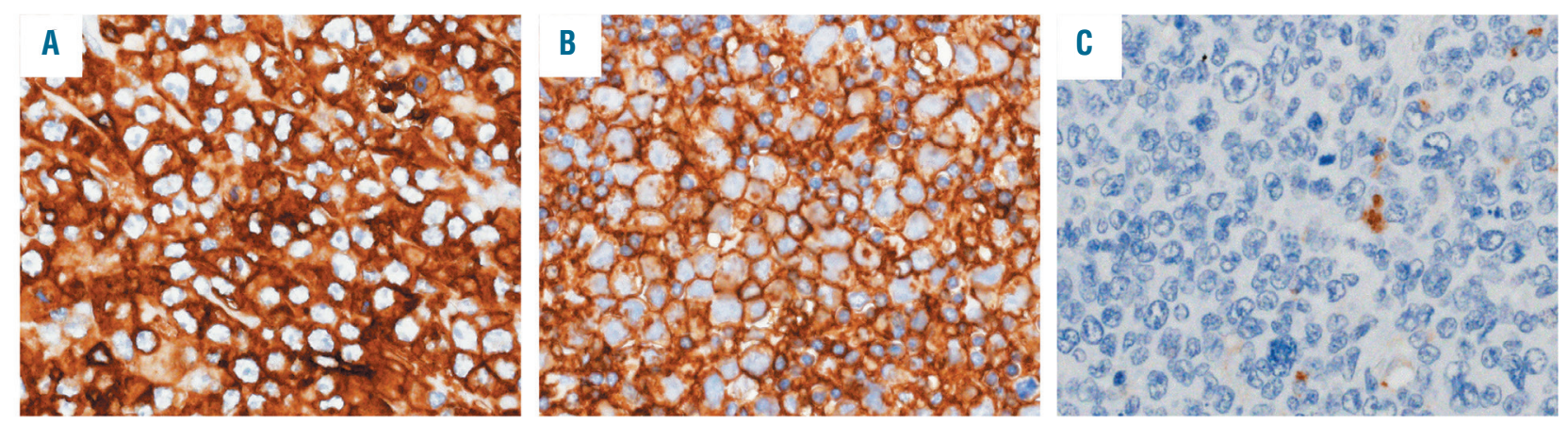

Figure 1. Immunohistochemistry of HLA class II in adult T-cell leukemia/lymphoma. (A, B) Representative images of human leukocyte antigen (HLA) class II-positive cases. Adult T-cell leukemia/lymphoma cells show membranes with/without cytoplasmic HLA class II expression. (C) A representative image of HLA class II-negative cases.

Table 1. Patients and treatment characteristics.

\begin{tabular}{lcc}
\hline Features & Numbers & $\%$ \\
Age, median (range), years & $67.5(35-90)$ & \\
Sex (male/female) & $75 / 57$ & \\
\hline PS score 2-4 & $43 / 129$ & 33.3 \\
IPI risk high or high-intermediate & $78 / 126$ & 61.9 \\
\hline JCOG-PI high & $50 / 129$ & 38.8 \\
Ann Arbor stage III or IV & $111 / 132$ & 84.1 \\
\hline Shimoyama classification & & \\
Acute type & $53 / 108$ & 49.1 \\
Lymphoma type & $52 / 108$ & 48.1 \\
Smoldering type & $3 / 108$ & 2.8 \\
Chronic type & $0 / 108$ & 0.0 \\
Treatment & & \\
Chemotherapy & $115 / 131$ & 87.8 \\
Radiation & $14 / 129$ & 10.9 \\
Transplantation & $17 / 129$ & 13.2 \\
No treatment & $12 / 130$ & 9.2 \\
\hline CR/CR(u) & $32 / 115$ & 27.8 \\
\hline PS: Perom & & \\
\hline
\end{tabular}

PS,: Performance Status; IPI: International Prognostic Index; JCOG-PI, Japan Clinical Oncology Group-Prognostic Index, CR: complete response; $\mathrm{CR}(\mathrm{u})$, : complete response (unconfirmed). Only the patients with available data were calculated.
Table 2. Immunohistochemical characteristics of patients wth adult Tcell leukemia/lymphoma.

\begin{tabular}{|c|c|c|}
\hline Features & Number & $\%$ \\
\hline 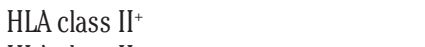 & $44 / 132$ & 33.3 \\
\hline HLA class II- & $88 / 132$ & 66.7 \\
\hline HLA class $\mathrm{I}^{\mathrm{m}+} \beta 2 \mathrm{M}^{\mathrm{m}+}$ & $48 / 120$ & 40.0 \\
\hline HLA class $\mathrm{I}^{\mathrm{m}}$-and/or $\beta 2 \mathrm{M}^{\mathrm{m}-}$ & $72 / 120$ & 60.0 \\
\hline HLA class $\mathrm{II}^{+} / \mathrm{HLA}$ class $\mathrm{I}^{\mathrm{m}}+\beta 2 \mathrm{M}^{\mathrm{m}+}$ & 22 & 18.3 \\
\hline HLA class $\mathrm{II}^{+} / \mathrm{HLA}$ class $\mathrm{I}^{\mathrm{m}}$-and/or $\beta 2 \mathrm{M}^{\mathrm{m}}$ & 17 & 14.2 \\
\hline HLA class II/HLA class $\mathrm{I}^{\mathrm{m}}+\beta 2 \mathrm{M}^{\mathrm{m}+}$ & 26 & 21.7 \\
\hline HLA class II/HLA class $\mathrm{I}^{\mathrm{m}}$-and/or $\beta 2 \mathrm{M}^{\mathrm{m}}$ & 55 & 45.8 \\
\hline nPD-L1+ & $9 / 130$ & 6.9 \\
\hline nPD-L1 & $121 / 130$ & 93.1 \\
\hline HLA class II $^{+}$nPD-L1+ & 5 & 3.8 \\
\hline HLA class II+ nPD-L1- & 39 & 30.0 \\
\hline HLA class II nPD-L1+ & 4 & 3.1 \\
\hline HLA class II nPD-L1- & 82 & 63.1 \\
\hline 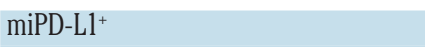 & $83 / 132$ & 62.9 \\
\hline miPD-L1 & $49 / 132$ & 37.1 \\
\hline $\mathrm{HLA}$ class $\mathrm{II}^{+} \mathrm{miPD}^{-\mathrm{Ll}^{+}}$ & 32 & 24.3 \\
\hline HLA class II $^{+}$miPD-L1 $^{-}$ & 12 & 9.1 \\
\hline HLA class II miPD-L1+ & 51 & 38.6 \\
\hline HLA class II miPD-L1' & 37 & 28.0 \\
\hline
\end{tabular}

ATLL;: Adult T-cell leukemia/lymphoma; HLA: human leukocyte antigen; $\beta 2 \mathrm{M}$ : $\beta 2$ microglobulin;nPD-L1:neoplastic programmed cell death ligand-1; miPD-L1:microenvironmental programmed cell death ligand-1.

Overall survival in patients with adult T-cell leukemia/lymphoma according to HLA class II expression

HLA class II $^{+}$cases had significantly better overall survival than HLA class II $^{-}$cases (median survival time, 36.53 months vs. 9.43 months, respectively; $P<0.0001$ ) (Figure 2).

Overall survival in patients with adult T-cell leukemia/Iymphoma according to HLA class II and HLA class I/ $\beta 2$-microglobulin expression

Compared to the other groups, HLA class $\mathrm{II}^{+} / \mathrm{HLA}$ class $\mathrm{I}^{\mathrm{m}+} / \beta 2 \mathrm{M}^{\mathrm{m}+}$ patients had the best clinical outcomes $(P=0.0013)$, although HLA class $\mathrm{II}^{+} /$HLA class $\mathrm{I}^{\mathrm{m}+} / \beta 2 \mathrm{M}^{\mathrm{m}+}$ patients also showed favorable outcomes (Figure 3). HLA class $\mathrm{II}^{-}$patients had a poor prognosis regardless of the HLA class I/ $/ 2 \mathrm{M}$ expression (Figure 3). 
Overall survival of patients with adult T-cell leukemia/lymphoma according to HLA class II and programmed death ligand-1 expression

As we have previously reported, the nPD-L1+ group had significantly worse overall survival than the nPD-L1group (median survival time, 5.93 months vs. 14.06 months, respectively; $P=0.0119) .{ }^{10}$ Moreover, miPD-L1 ${ }^{+}$ patients had a better prognosis than miPD-L1- patients (median survival time 15.63 months vs. 10.1 months, respectively) $(P=0.0029) .{ }^{10}$

Patients in the HLA class II $/ \mathrm{nPD}-\mathrm{L} 1$ group had a significantly better survival time than the other patients. $(P$ $<0.0001$ ) (Figure $4 \mathrm{~A}$ ). The HLA class $\mathrm{II}^{+} / \mathrm{miPD}^{-L} 1^{+}$group had significantly better overall survival than the other groups $(P<0.0001)$ (Figure $4 \mathrm{~B})$.

\section{Clinico-pathological differences between HLA \\ class II-positive and HLA class II-negative cases}

The clinicopathological characteristics of HLA class $\mathrm{II}^{+}$ and HLA class II $^{-}$cases are summarized in Table 3. HLA class $\mathrm{II}^{+}$cases showed a significantly higher complete response rate $(P=0.0421)$, higher HLA class $\mathrm{I}^{+} / \beta 2 \mathrm{M}^{+}$ expression $(P=0.0165)$, and increased number of $P D-1^{+}$ tumor-infiltrating lymphocytes $(P=0.0020)$. Performance Status, International Prognostic Index, JCOG-PI, clinical stage, and therapies were not significantly different between the two groups. The association of HLA class II, PD-L1, and PD-1 expression is shown in Online Supplementary Figure S2. There was no significant association among them.

\section{Prognostic factors in patients with adult T-cell leukemia/lymphoma}

Table 4 presents results of univariate and multivariate analyses of prognostic factors in ATLL. In univariate analyses, age (>70 years) [hazard ratio (HR) 1.709; 95\% confidence interval (95\% CI): 1.106-2.608; $P=0.0164]$, high JCOG-PI (HR 1.841; 95\% CI: 1.186-2.819; $P=0.0071$ ), Ann Arbor stage III or IV (HR 1.801; 95\% CI: 1.002-3.583; $P=0.0491$ ), HLA class II expression (HR 0.386; 95\% CI: $0.233-0.617 ; P<0.0001$ ), and HLA class II/miPD-L1 expression (HR 0.276; 95\% CI: 0.146-0.482; $P<0.0001$ ) were significantly related to prognosis.

In a multivariate analysis of HLA class II expression and other factors, HLA class II expression (HR 0.441; 95\% CI: $0.263-0.714 ; P=0.0007$ ) and high JCOG-PI (HR 1.841; $95 \%$ CI: 1.163-2.883; $P=0.0096$ ) showed significant prognostic value.

We also performed a random forest analysis including all available clinical prognostic factors (JCOG-PI, age $>70$ years, elevated lactate dehydrogenase, extranodal involvement, sex, elevated C-reactive protein, B-symptoms, clinical stage, chemotherapy, radiation, and transplantation) and HLA class II expression. JCOG-PI, HLA class II expression, and age were associated with patients living for more than 2 years.

\section{Discussion}

In this report, we describe that HLA class II expression is an important prognostic factor in ATLL, as are both HLA class $\mathrm{I} / \beta 2 \mathrm{M}$ and $\mathrm{PD}-\mathrm{L} 1$ expression. HLA class II had a prognostic value in ATLL patients treated with standard chemotherapy in our study. Malignant melanoma,
Hodgkin lymphoma and lung cancer are often associated with PD-L1 expression on tumor cells and respond to immune checkpoint inhibitors. However, HLA class II was not associated with prognosis in patients with these cancers treated with standard chemotherapy. ${ }^{23-25}$ In our study ATLL cases co-expressing PD-L1 and HLA class II, had a very poor prognosis, although the number of patients analyzed was small ( $n=5,3.8 \%$ ). In the cases of Hodgkin lymphoma and malignant melanoma, HLA class II expression predicted the clinical outcome of PD-1 blockade therapy, suggesting that the prediction of prognosis by HLA class II expression depends on the expression of PD-L1. ${ }^{24,26}$

HLA class $\mathrm{I} / \beta 2 \mathrm{M}$ is also a good prognostic marker in ATLL patients. However, HLA class $\mathrm{I}^{+} / \beta 2 \mathrm{M}^{+}$patients without HLA class II expression had a poor prognosis in this

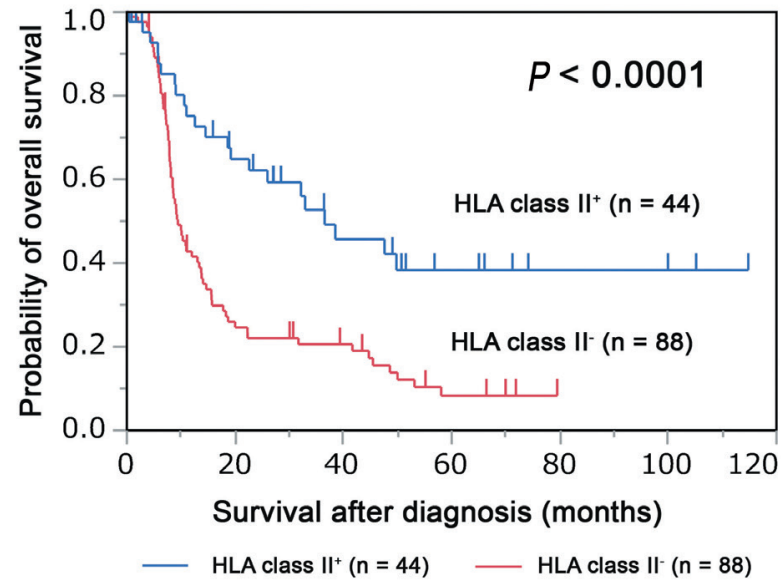

Figure 2. Association of HLA class II expression with overall survival in adult Tcell leukemia/lymphoma. The Kaplan-Meier plot depicts the survival difference between human leukocyte antigen (HLA) class II-positive (HLA class $\mathrm{II}^{+}$) and HLA class II-negative (HLA class II-) patients ( $P<0.0001$; log-rank test).

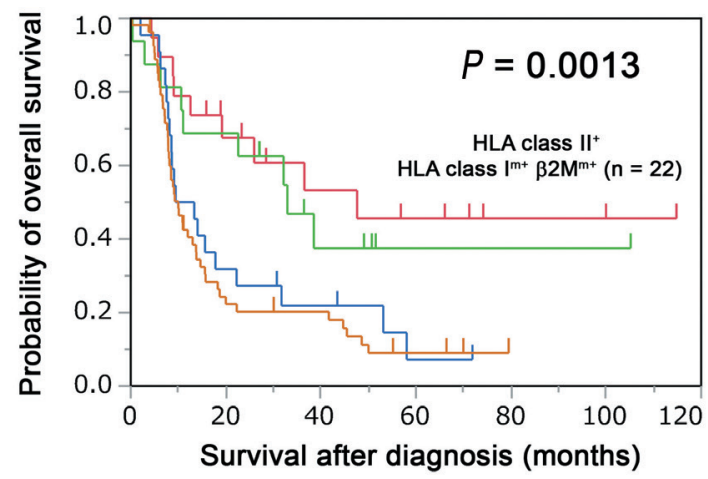

- HLA class $\mathrm{II}^{+}$HLA class $\mathrm{I}^{m+} \beta 2 \mathrm{M}^{m+}(n=22) \longrightarrow$ HLA class $\mathrm{II}^{+}$HLA class $\mathrm{I}^{\mathrm{m}-}$ and/or $\beta 2 \mathrm{M}^{m-}(n=17)$

- HLA class II HLA class $\mathrm{I}^{m+} \beta 2 \mathrm{M}^{m+}(n=26)$

- HLA class II HLA class I $^{m}$ and/or $\beta 2 M^{m-}(n=55)$

Figure 3. Overall survival according to expression of HLA class II and HLA class I/ $\beta 2$ microglobulin in patients with adult T-cell leukemia/lymphoma. The patients with adult T-cell leukemia/lymphoma were stratified into four groups according to human leukocyte antigen (HLA) class II and HLA class $1 / \beta 2$ microglobulin $(\beta 2 \mathrm{M})$ expression. Differences in overall survival among the four groups are shown in the Kaplan-Meier plot $(P=0.0013$; log-rank test). 
study. Recently, the importance of $\mathrm{CD}^{+} \mathrm{T}$ cells and HLA class II-restricted neoantigens has been focused on in cancer immunology. It has been suggested that $\mathrm{CD} 4^{+}$type 1 helper T cells can upregulate HLA class II expression on tumor cells by producing cytokines and that they can kill tumor cells directly, independently of other immune cells. ${ }^{30}$ Thus, $\mathrm{CD}^{+}$non-neoplastic $\mathrm{T}$ cells may also contribute to the improved prognosis of HLA class $\mathrm{II}^{+}$ATLL patients. In addition, $\mathrm{CD}^{+}$helper $\mathrm{T}$ cells can help $\mathrm{CD}^{+} \mathrm{T}$ cells in tumor immunity. ${ }^{30}$ Thus, HLA class II expression may be necessary for $\mathrm{CD}^{+} \mathrm{T}$ cells to attack HLA class $\mathrm{I}^{+}$ tumor cells.

HLA class II expression was also reported to be a good prognostic factor in diffuse large B-cell lymphoma, ${ }^{22}$ a malignancy derived from B cells. Thus, diffuse large B-cell lymphoma originally has active HLA class II, which can present neoantigens, suggesting that loss of HLA class II on diffuse large B-cell lymphoma disrupts the interaction with $\mathrm{CD}^{+} \mathrm{T}$ cells.

In our study, PD-L1-expressing stromal cells (miPD-L1+)
A

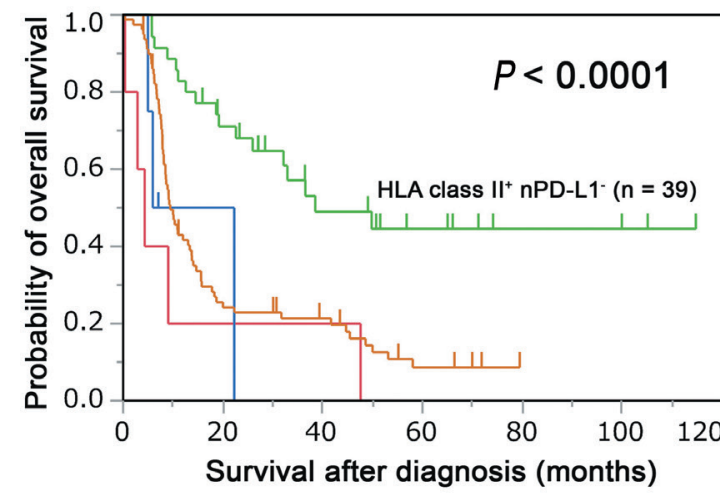

HLA class $\mathrm{II}^{+}$nPD-L1- $(\mathrm{n}=39)$

- HLA class II+ nPD-L1+ $(n=5)$
- HLA class II- nPD-L1 $1^{+}(n=4)$
- HLA class II- nPD-L1 $(n=82)$
B

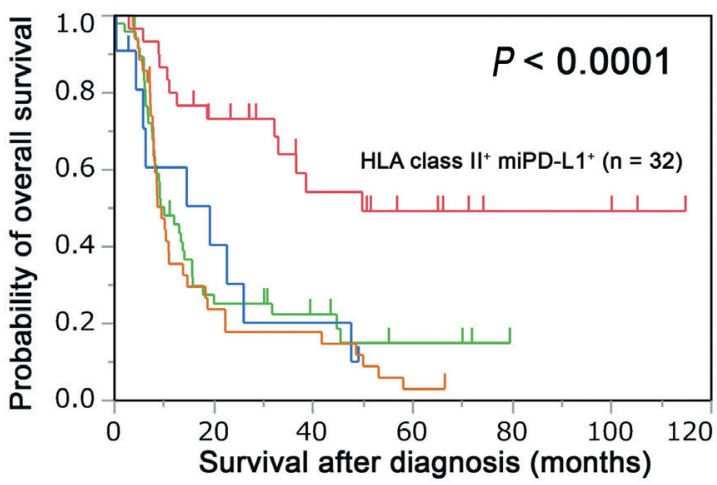

- HLA class $\mathrm{II}^{+}$miPD-L1+ $(n=32)-$ HLA class $\mathrm{II}^{+}$miPD-L1 $(n=12)$

HLA class II- miPD-L1+ $(n=51)$

HLA class II- miPD-L1- $(n=37)$

Figure 4. Overall survival associated with HLA class II and programmed death ligand-1 expression. (A) Patients were stratified into four groups according to the expression of human leukocyte antigen (HLA) class II and programmed death ligand-1 on neoplastic cells (nPD-L1). The differences in survival are depicted in the Kaplan-Meier plot $(P<0.0001$; log-rank test). (B) The patients were divided into four groups according to the expression of HLA class II and programmed death ligand-1 on microenvironmental cells (miPD-L1). Kaplan-Meier plots reveal the differences among the groups $(P<0.0001$; log-rank test).

Table 3. Clinicopathological comparison according to HLA class II expression.

\begin{tabular}{|c|c|c|c|c|c|}
\hline I & HLA class II+ $(n=44)$ & $\%$ & HLA class II' (n=88) & $\%$ & $P$ \\
\hline Age, mean (range), years & $65.5(35-80)$ & & $68.5(40-90)$ & & 0.0697 \\
\hline Sex, male/female & $28 / 16$ & & $47 / 41$ & & 0.3515 \\
\hline \multicolumn{6}{|l|}{ Shimoyama classification } \\
\hline Acute type & $19 / 35$ & 54.3 & $34 / 73$ & 46.6 & 0.5387 \\
\hline Lymphoma type & $15 / 35$ & 42.8 & $37 / 73$ & 50.7 & 0.5382 \\
\hline Smoldering type & $1 / 35$ & 2.9 & $2 / 73$ & 2.7 & - \\
\hline Chronic type & $0 / 35$ & 0.0 & $0 / 73$ & 0.0 & - \\
\hline PS score 2-4 & $12 / 44$ & 27.3 & $31 / 85$ & 36.5 & 0.3297 \\
\hline IPI high or high-intermediate & $25 / 43$ & 58.1 & $53 / 83$ & 63.9 & 0.5654 \\
\hline JCOG-PI high & $14 / 44$ & 31.8 & $36 / 85$ & 42.4 & 0.2601 \\
\hline Ann Arbor stage III, IV & $34 / 44$ & 77.3 & $77 / 88$ & 87.5 & 0.1387 \\
\hline Elevated LDH & $27 / 44$ & 61.4 & $49 / 88$ & 55.7 & 0.5788 \\
\hline \multicolumn{6}{|l|}{ Treatment } \\
\hline Chemotherapy & $36 / 44$ & 81.8 & $79 / 87$ & 90.8 & 0.1624 \\
\hline Radiation & $4 / 43$ & 9.3 & $10 / 86$ & 11.6 & 0.7731 \\
\hline Transplantation & $4 / 43$ & 9.3 & $13 / 86$ & 15.1 & 0.4205 \\
\hline No treatment & $7 / 44$ & 15.9 & $5 / 86$ & 5.8 & 0.1049 \\
\hline $\mathrm{CR} / \mathrm{CR}(\mathrm{u})$ & $15 / 36$ & 41.7 & $17 / 79$ & 21.5 & $0.0421^{*}$ \\
\hline \multicolumn{6}{|l|}{ Immunohistochemistry } \\
\hline HLA class $I^{\mathrm{m}+} \beta 2 \mathrm{M}^{\mathrm{m}+}$ & $22 / 39$ & 56.4 & $26 / 81$ & 32.1 & $0.0165^{*}$ \\
\hline $\mathrm{nPD}-\mathrm{L1}^{+}$ & $5 / 44$ & 11.4 & $4 / 86$ & 4.7 & 0.1655 \\
\hline miPD-L1+ & $32 / 44$ & 72.7 & $51 / 88$ & 58.0 & 0.1266 \\
\hline PD-1+ TIL (hpf), average (range) & $3.95(0-37.4)$ & & $1.83(0-38.4)$ & & $0.0020^{*}$ \\
\hline
\end{tabular}

HLA: human leukocyte antigen; PS: Performance Status; IPI: International Prognostic Index; JCOG-PI; Japan Clinical Oncology - Prognostic Index; LDH: lactate dehydrogenase; CR: complete response; CR(u),: complete response (unconfirmed); $\beta 2 \mathrm{M}$ : $\beta 2$ microglobulin; nPD-L1: neoplastic programmed death ligand-1; miPD-L1: microenvironmental programmed death ligand-1; PD-1: programmed death-1;TIL: tumor infiltrating cells; hpf: high power field. *Statistically significant $P$ value. 
Table 4. Prognostic factors associated with overall survival in patients with adult T-cell leukemia/lymphoma.

\begin{tabular}{|c|c|c|c|c|}
\hline \multirow[b]{2}{*}{ Characteristics } & \multicolumn{2}{|c|}{ Univariate analysis } & \multicolumn{2}{|c|}{ Multivariate analysis } \\
\hline & HR (95\% CI) & $\boldsymbol{P}$ & HR (95\% CI) & $P$ \\
\hline HLA class $\mathrm{II}^{+}$ & $0.386(0.233-0.617)$ & $<0.0001^{*}$ & $0.441(0.263-0.714)$ & $0.0007^{*}$ \\
\hline HLA class II+/miPD-L1+ & $0.276(0.146-0.482)$ & $<0.0001^{*}$ & & \\
\hline JCOG-PI (high) & $1.841(1.186-2.819)$ & $0.0071^{*}$ & $1.841(1.163-2.883)$ & $0.0096 *$ \\
\hline Age $>70$ years & $1.709(1.106-2.608)$ & 0.0164 & $1.441(0.925-2.217)$ & 0.1048 \\
\hline Ann Arbor stage III, IV & $1.801(1.002-3.583)$ & $0.0491^{*}$ & $1.661(0.915-3.325)$ & 0.0982 \\
\hline Elevated LDH (>normal) & $0.982(0.651-1.492)$ & 0.9344 & $0.934(0.605-1,447)$ & 0.7582 \\
\hline
\end{tabular}

ATLL: adult T-cell leukemia/lymphoma; HR: hazard ratio; 95\% CI: 95\% confidence interval; HLA: human leukocyte antigen; miPD-L1: microenvironmental programmed cell death ligand-1; JCOG-PI: Japan Clinical Oncology Group-Prognostic Index; LDH: lactate dehydrogenase.. * Statistically significant $P$ value.

seemed necessary for good clinical outcome of HLA class $\mathrm{II}^{+}$patients. miPD-L1 has been reported in some cancers, but the precise role of miPD-L1 is unclear. It is possible that miPD-L1 suppressed PD-1-expressing tumor cells, but no significant association between miPD-L1 and neoplastic PD-1 expression was observed in the present study (data not shown). Further research is required to determine whether there are direct or indirect interactions between HLA class II $^{+}$tumor cells and miPD-L1.

Ratner et al. recently reported rapid progression of ATLL after PD-1 blockade therapy. ${ }^{27}$ They attributed the disease progression to the tumor suppressive role of $\mathrm{PD}-1$ in ATLL. It seems that the ATLL patients included in their study expressed PD-L1 on only $<1 \%$ or $5 \%$ of tumor cells. Our current and previous data suggest that expression of HLA class I/class II and miPD-L1 can predict good clinical outcome in ATLL patients without massive nPD-L1 expression. HLA class I/class II expression might be associated with the efficacy of PD-1 blockade therapy in ATLL. Although the precise function of miPD-L1 remains to be determined, PD-1 blockade might disrupt tumor immunity associated with miPD-L1 in ATLL.

HLA class II expression on activated normal human $T$ cells is regulated by class II transactivator (CIITA). ${ }^{31}$ In Tcell malignancies, expression of CIITA also correlates with HLA class II expression. ${ }^{31}$ In ATLL, CIITA was reported to inhibit the nuclear factor $\kappa \mathrm{B}$ pathway activated by the Tax1 oncoprotein of HTLV-1. ${ }^{32}$ Thus, the favorable clinical prognosis of HLA class $\mathrm{II}^{+}$ATLL patients may correlate with upregulation of CIITA, which leads to activation of HLA class II expression as well as suppression of the NF$\kappa \mathrm{B}$ signaling pathway.

The rate of HLA class II positivity was low in our study compared to that in the published literature, in which chronic and smoldering ATLL were reported to show high expression of HLA class II whereas acute type ATLL was described as showing low expression of HLA class II ${ }^{33}$ It was suggested that HLA class II expression on ATLL could be associated with progression of chronic ATLL. ${ }^{33}$ Our study included mostly patients with acute or lymphoma type ATLL. HLA class II expression on acute or lymphoma type ATLL may be correlated with better clinical outcome.

In conclusion, we report, for the first time, that HLA class II expression is a good prognostic marker in ATLL. Both HLA class II and miPD-L1 were required for good clinical outcome. Our results help to understand tumor immunity in ATLL.

\section{Acknowledgments}

The authors thank Kazutaka Nakashima, Mayumi Miura, Kanoko Miyazaki, and Chie Kuroki for their technical assistance and collaborators from the following institutions for providing clinical data and specimens to the Kyushu Lymphoma Study Group: Department of Medicine and Biosystemic Science, Kyushu University Faculty of Medicine; Department of Hematology, Karatsu Red Cross Hospital; Department of Hematology, Sasebo City General Hospital; Department of Hematology, Atomic Bomb Disease and Hibakusha Medicine Unit, Atomic Bomb Disease Institute, Nagasaki University; and Department of Medicine and Bioregulatory Science, Graduate School of Medical Sciences, Kyushu University. This work was supported in part by Grantsin-Aid from the Japan Agency for Medical Research and Development (15ck0106015h0002) (MS); the Japan Society for the Promotion of Science (KAKENHI), grant numbers JP26460446 (KO) and JP17K17894 (MT); and the Japan Leukemia Research Fund (General Research Award 2013) (NA). The language and format of this manuscript were edited by Editage (https://www.editage.com).

\section{References}

1. Ohshima K, Jaffe E, Yoshino T, Siebert R. Adult T-cell leukemia/lymphoma. In Swerdlow SH, Campo CE, Harris NL, et al. eds. World Health Organization Classification of Tumours. Revised 4th ed. Lyon, France: IARC Press, 2017;363-367.

2. Tajima K, Hinuma Y. Epidemiology of HTLV-I/II in Japan and the world. In Takatsuki K, Hinuma Y, Yoshida M eds. Advances in Adult T-cell Leukemia and
HTLV-I Research. Tokyo: Japan Scientific Societies Press, 1992;129-149.

3. Shimoyama M. Diagnostic criteria and classification of clinical subtypes of adult T-cell leukaemia-lymphoma. A report from the Lymphoma Study Group (1984-87). Br J Haematol. 1991;79(3):428-437.

4. Takasaki Y, Iwanaga M, Imaizumi Y, et al. Long-term study of indolent adult T-cell leukemia-lymphoma. Blood. 2010;115(22); 4337-4343

5. Gao Q, Wang XY, Qiu SJ, et al.
Overexpression of PD-L1 significantly associates with tumor aggressiveness and postoperative recurrence in human hepatocellular carcinoma. Clin Cancer Res. 2009;15(3):971-979.

6. Nomi T, Sho M, Akahori T, et al. Clinica significance and therapeutic potential of the programmed death-1 ligand/programmed death-1 pathway in human pancreatic cancer. Clin Cancer Res. 2007;13(7):2151-2157.

7. Ohigashi Y, Sho M, Yamada Y, et al. Clinical significance of programmed death-1 ligand- 
1 and programmed death-1 ligand-2 expression in human esophageal cancer. Clin Cancer Res. 2005;11(8):2947-2953.

8. Thompson $\mathrm{RH}$, Dong $\mathrm{H}$, Kwon ED. Implications of $\mathrm{B} 7-\mathrm{H} 1$ expression in clear cell carcinoma of the kidney for prognostication and therapy. Clin Cancer Res. 2007;13(2);709-715.

9. Zou W, Chen L. Inhibitory B7-family molecules in the tumour microenvironment. Nat Rev Immunol. 2008;8(6):467-477.

10. Miyoshi H, Kiyasu J, Kato T, et al. PD-L1 expression on neoplastic or stromal cells is respectively a poor or good prognostic factor for adult T-cell leukemia/lymphoma. Blood. 2016;128(10):1374-1381.

11. Hicklin DJ, Wang Z, Arienti F, et al. $\beta 2$ microglobulin mutations, HLA class I antigen loss, and tumor progression in melanoma. J Clin Invest. 1998;101(12);2720-2729.

12. Kataoka K, Nagata Y, Kitanaka A, et al. Integrated molecular analysis of adult $\mathrm{T}$ cell leukemia/lymphoma. Nat Genet. 2015;47 (11):1304-1315

13. Asano N, Miyoshi $H$, Kato $T$, et al. Expression pattern of immunosurveillancerelated antigen in adult $\mathrm{T}$ cell leukaemia/ lymphoma. Histopathology. 2018;72(6): 945-954.

14. Keir ME, Francisco LM, Sharpe AH. PD-1 and its ligands in T-cell immunity. Curr Opin Immunol. 2007;9(3):309-314.

15. Pedoeem A, Azoulay-Alfaguter I, Strazza M, et al. Programmed death-1 pathway in cancer and autoimmunity. Clin Immunol. 2014;153(1):145-152.

16. Melief CMJ. Precision T-cell therapy targets tumours. Nature. 2017;547(7662):217-222.

17. Seliger B. Molecular mechanisms of HLA class I-mediated immune evasion of human tumors and their role in resistance to immunotherapies. HLA. 2016;88(5): 213220
18. Ansell SM, Lesokhin AM, Borrello I, et al. PD-1 blockade with nivolumab in relapsed or refractory Hodgkin's lymphoma. N Engl Med. 2015; 372(4):311-319.

19. Reichel J, Chadbum A, Rubunstein PG, et al Flow sorting and exome sequencing reveal the oncogene of primary Hodgkin and ReedSternberg cells. Blood. 2015;125(7):10611072.

20. Sconocchia G, Eppenberger-Castori S, Zlobec I, et al. HLA class II antigen expression in colorectal carcinoma tumors as a favorable prognostic marker. Neoplasia. 2014;16(1):31-42

21. Esteban F, Ruiz-Cabello F, Concha A, et al HLA-DR expression is associated with excellent prognosis in squamous cell carcinoma of the larynx. Clin Exp Metastasis. 1990;8(4):319-328.

22. Rimsza LM, Robberts RA, Campo E, et al. Loss of MHC class II gene and protein expression in diffuse large B-cell lymphoma is related to decreased tumor immunosurveillance and poor patient survival regardless of other prognostic factors: a follow-up study from the Leukemia and Lymphoma Molecular Profiling Project. Blood. 2004:103(11):4251-4258.

23. He Y, Rozeboomb L, Rivardb CJ, et al. MHC class II expression in lung cancer. Lung Cancer. 2017;112:75-80.

24. Johnson DB, Estrada MV, Salgado R, et al. Melanoma-specific MHC-II expression represents a tumour-autonomous phenotype and predicts response to anti-PD1/PD-L1 therapy. Nat Commun. 2016;29 (7):10582.

25. Roemer MG, Advani RH, Redd RA, et al Classical Hodgkin lymphoma with reduced $\beta 2 \mathrm{M} / \mathrm{MHC}$ class I expression is associated with inferior outcome independent of 9p24.1 status. Cancer Immunol Res. 2016;4(11):910-916.
26. Roemer MGM, Redd RA, Cader FZ, et al. Major histocompatibility complex class II and programmed death ligand expression predict outcome after programmed death 1 blockade in classic Hodgkin lymphoma. J Clin Oncol. 2018; 36(10):942-950.

27. Ratner L, Waldman TA, Janakiram $M$, et al. Rapid progression of adult T-cell leukemialymphoma after PD-1 inhibitor therapy. N Engl J Med. 2018;378(20):1947-1948.

28. Weisenburger DD1, Savage KJ, Harris NL, et al. International Peripheral T-cell Lymphoma Project. Peripheral T-cell lymphoma, not otherwise specified: a report of 340 cases from the International Peripheral T-cell Lymphoma Project. Blood. 2011;117(12): 3402-3408.

29. Fukushima T, Nomura S, Shimoyama M, et al. Japan Clinical Oncology Group (JCOG) prognostic index and characterization of long term survivors of aggressive adult $\mathrm{T}$ cell leukaemia lymphoma (JCOG0902A). Br J Haematol. 2014;166(5):739-748.

30. Sun Z. Chen F, Meng F, Wei J, Liu B. MHC class II restricted neoantigen: a promising target in tumor immunotherapy. Cancer Let. 2017;392:17-25.

31. Holling TM, Schooten E, Langerak AW, van den Elsen PJ. Regulation of MHC class II expression in human T-cell malignancies. Blood. 2004;103(4):1438-1444.

32. Forlani G, Abdallah R, Accol RS, Tosi G. The major histocompatibility complex class II transactivator CIITA inhibits the persistent activation of NF- $\mathrm{KB}$ by the human $\mathrm{T}$ cell lymphptropic virus type 1 Tax-1 oncoprotein. J Virol. 2016;90(7):3708-3721.

33. Shirono K, Hattori T, Hata H, Nishimura $H$ Takatsuki K. Profiles of expression of activated cell antigens on perioheral blood and lymph node cells from different clinical stages of sdult T-cell leukemia. Blood. 1989;73(6):1664-1671. 